\title{
EVALUATION OF TEN BARLEY GENOTYPES UNDER NORMAL IRRIGATION AND WATER STRESS CONDITIONS
}

\author{
E. H. El-Seidy ${ }^{(1)}$, E. E. EL-Shawy ${ }^{(2)}$, U.A. Abd El Razek ${ }^{(1)}$ \\ and W. G. Alkordi ${ }^{(1)}$ \\ (1) Agronomy Dept., Fac. of Agric., Tanta University \\ (2) Barley Dept., Field Crops Res. Sakha, ARC, Egypt
}

Received: Aug. 8, 2017

Accepted: Aug. 14, 2017

\begin{abstract}
This research was conducted in order to identify the best barley genotypes that can be grown under water stress conditions. This experiment was conducted in a split plot design with three replications at Experimental Farm of Sakha Agricultural Research Station, (ARC), Egypt, during the two successive seasons 2014/15 and 2015/16. Ten barley genotypes (Giza 123, Giza 126, Giza 129, Giza 130, Giza 131, Giza 132, Giza 133, Giza 134, Giza 135 and Giza 136) were evaluated for drought tolerance by measuring yield performance under normal irrigation and water stress conditions. The results indicated that, all the studied characters were significantly affected by stress in both growing seasons, except for total chlorophyll, spike length, no of grains/spike and harvest index, in both seasons. Stress reduced grain yield (ardab/fad.) by reducing the number of spikes $/ \mathrm{m}^{2}$ and 1000-kernel weight. This study showed that, the best genotypes of barley for all parameters studied under stress condition were Giza 133 followed by Giza 134 in both seasons.
\end{abstract}

Key words: Water Stress - Drought tolerance-Evaluations - Barely.

\section{INTRODUCTION}

Barley (Hordeum vulgare L.) is the fourth grain crop both in area and production in the world after maize, wheat and rice. It has the potential to become one of the important cereal crops in Egypt (El-Shawy, 2013 and El-Shawy, et al., 2013). The rainfed areas in Egypt cover about 120,000 hectares in the North West Coast and about 40.000 hectares in NorthSinai. Farming systems of these populations are livestock mainly sheep with barley as their main annual crop for fodder and breadmaking (El-Shawy, et al., 2013).

Drought is a major abiotic stress that severely affects barley production worldwide. Therefore, research into crop management practices that enhance drought tolerance and plant growth when water supply is limited has become increasingly essential. Barley germplasm is a treasure trove of useful genes and provide rich sources of genetic variation for crop improvement.
Drought resistance in crops is probably the most difficult trait to understand (Ashraf, 2010) due to lack of comprehensive information regarding the genetic mechanism of drought tolerance and grain yield under drought conditions (Farashdfar and Sutka, 2002).

The objectives of this study, therefore, were to screen barley genotypes with high yield under water stress conditions.

\section{MATERIALS AND METHODS}

Ten barley genotypes (Giza 123, Giza 126, Giza 129, Giza 130, Giza 131, Giza 132, Giza 133, Giza 134, Giza 135 and Giza 136) were chosen for the study based on their reputed differences in yield performance under normal and stress conditions. Experiments were conducted at the Experimental Farm of Sakha Agricultural Research Station, (ARC), Egypt, during the two successive seasons 2014/15 and 2015/16. 
Soil samples were randomly taken from the experimental area at a depth of 0 to $30 \mathrm{~cm}$ from soil surface before barley sowing. The soil properties are shown in Table 1.

In the first season, the maximum temperature was high and the relative humidity and rainfall were low compared with the second season (Table 2).

This experiment was laid out in split plot design with three replications, two methods of irrigation (normal and water stress condition (sowing irrigation only) plotted in main plots and ten barely genotypes (Giza 123, Giza 126, Giza 129, Giza 130, Giza 131, Giza 132, Giza 133, Giza 134, Giza 135 and Giza 136) were tried in a sub-plot. Grains were hand drilled at the recommended sowing rate of barley in the irrigated land in Egypt (50 kg fed.-1). Each genotype was sown in six rows of $3.5 \mathrm{~m}$, spaced with $20 \mathrm{~cm}$ among rows. The normal irrigation treatment were irrigated twice after sowing, at 45 days after sowing at tillering stage and 75 days after sowing at booting stage (normal condition), while, the drought irrigation treatments were given just sowing irrigation only (drought stress condition). Sowing was done in 15th of November in both seasons. All recommended culture practices were applied at proper time according to ministry of agriculture recommended. The preceding crop was cotton in the two seasons (Table $3)$.

Data were subjected to the proper statistical analysis as the technique of analysis of variance (ANOVA) of split plot design as mentioned by Gomez and Gomez (1984). Treatment means were compared using the Least Significant Difference (LSD) test as outlined by Waller and Duncan (1969).

The following data was recorded during the growing seasons and after harvesting time as follows:

Table (1): Soil analysis of the Experimental Field at Sakha Agricultural Research Station at 2014/15 and 2015/16 Seasons .

\begin{tabular}{|c|c|c|c|c|c|c|}
\hline Determination & Sand \% & Silt \% & Clay \% & Texture & $\mathrm{pH}$ & $\mathrm{E} . \mathrm{C}(\mathrm{ds} / \mathrm{m})$ \\
\hline $1^{\text {st }}$ Season & 13.74 & 24.91 & 61.35 & Clay & 7.9 & 2.1 \\
\hline $2^{\text {nd }}$ Season & 15.53 & 23.95 & 60.52 & Clay & 8.2 & 2.9 \\
\hline
\end{tabular}

In the first season, the maximum temperature was high and the relative humidity and rainfall were low compared with the second season (Table 2). Ten barley genotypes were used, their names, pedigrees and origin are presented in Table 3.

Table (2): Maximum, minimum temperature and rainfall during the growing seasons of barley crop at Sakha Agricultural Research Station, (ARC), Egypt.

\begin{tabular}{|c|c|c|c|c|c|c|}
\hline \multirow{3}{*}{ Month } & \multicolumn{4}{|c|}{ Temperature $\left(\mathrm{C}^{\circ}\right)$} & \multicolumn{2}{c|}{ Rainfall $(\mathrm{mm})$} \\
\cline { 2 - 5 } & \multicolumn{2}{|c|}{$2014 / 15$} & \multicolumn{2}{c|}{$2015 / 16$} & \multicolumn{2}{c|}{} \\
\cline { 2 - 7 } & Max. & Min. & Max. & Min. & $2014 / 15$ & $2015 / 16$ \\
\hline Dec. & 20 & 13 & 19 & 11 & 15 & 27 \\
\hline Jun. & 17 & 12 & 18 & 9 & 12 & 42 \\
\hline Feb. & 19 & 14 & 22 & 11 & 5 & 14 \\
\hline Mar. & 21 & 15 & 23 & 13 & 15 & 6 \\
\hline Apr. & 22 & 17 & 24 & 14 & -- & -- \\
\hline May. & 25 & 19 & 26 & 17 & -- & -- \\
\hline
\end{tabular}


Evaluation of ten barley genotypes under normal and drought stress

Table (3): Name and pedigree of ten barley genotypes.

\begin{tabular}{|c|c|c|}
\hline Genotypes & NamelCross & Origin \\
\hline Giza 123 & Giza 117//FAO86 & Egypt \\
\hline Giza 126 & Baladi Bahteem/SD729-por12762-Bc & Egypt \\
\hline Giza 129 & Deir Alla 106/Cel//As46/Aths*2 & Egypt \\
\hline Giza 130 & "Comp.cross"229//Bco.Mr./DZ0231/3 /Deir Alla106 & Egypt \\
\hline Giza 131 & $\begin{array}{l}\text { CM67-B/CENTENO//CAM- B /3/ ROW906.73 /4 / GLORIA- } \\
\text { BAR/COME-B/5/ FALCON -BAR /6/ LINO }\end{array}$ & Egypt \\
\hline Giza 132 & Rihane-05//As46/Aths*2" Aths/ Lignee686 & Egypt \\
\hline Giza 133 & Carbo/Gustoe & Egypt \\
\hline Giza 134 & Alanda-01/4/WI2291/3/Api/CM67//L2966-69 & Egypt \\
\hline Giza 135 & Zarza/Bermejo/4/DS4931//Gloria-Bar/Copal/3/Sen/5/Ayarosa" & Egypt \\
\hline Giza 136 & $\begin{array}{l}\text { Plaisant/7/CLN-B/4/S.P-B/Lingee640/3/S.P-B// Gloria-BAR/Come- } \\
\text { B/5/Falcon-BAR/6/Lion/CLN-B/A/S.P-B/Lingee640/3/S.P- } \\
\text { B//Gloria-BAR/Come-B/5/Falcon-BAR /6/ Lino }\end{array}$ & Egypt \\
\hline
\end{tabular}

\section{Data recorded}

1-Total chlorophyll content: was determined by measuring the flag leaf total chlorophyll content by using analytical apparatus; chlorophyll meter (Model SPAD- 502) Minolta camera Co. Ltd, Japan.
2- Heading date
3- Maturity date
4- Plant height
5 -Number of spikes $/ \mathrm{m}^{2}$
6- Spike length 7- Number of grains/spike
8-1000-grain weight $(\mathrm{g})$
9- Biological yield (ton/fed.)
10- Grain yield (ton/fed.) 11-Harvest index

\section{RESULTS AND DISCUSSION Effect of irrigation treatments:}

The results in Table (4 a and b) indicated that, all the studied characters were significantly affected by water stress in both growing seasons, except for total chlorophyll, spike length, no of grains/spike and harvest index in both seasons, heading date in the second season and 1000-grain weight in the first season. The results showed that, the non-stress irrigation resulted in higher values for all the studies characters compared with the stress irrigation in both seasons, except total chlorophyll and harvest index.

A severe reduction in plant height is a common type of plant response to water stress in barley as reported by Ceccarelli (1986). These results are in line with those obtained by El-Seidy (1997), Gaspar et al., (1998), El-Madidi et al., (2005) and Abu-ElLail et al., (2016).

The application of severe stress decreased grain weight and this may be due to water stress which reduced the final grain weight by curtailing the duration of the grain filling stage. Moisture stress applied just before or during the maturity process greatly reduced seed weight (Robins and Domingo, 1962). These results were in accordance with those of Assey et al., (1990), Samarah (2005) and Abu-El-Lail et al., (2016). 
E. H. El-Seidy, et al.,

The yield reduction was much more severe if water stress occurred during and following heading, resulting in fewer heads, fewer spikelets /spike, and fewer kernels per spike (Robins and Domingo, 1962). Severe drought stress at $20 \%$ field capacity until grain maturity reduced grain yield by reducing the number of tillers, spikes and grains per plant and individual grain weight (Samarah, 2005). These results go in line with those obtained by Kheiralla et al. (1997), Tarred et al., (2002), Motawei and Abdalla (2003), El-Kholy et al., (2005), Karami et al. (2005) and Abu-El-Lail et al., (2016).

Table (4 a): Effect of irrigation treatments on barley characters in both growing seasons

\begin{tabular}{|c|c|c|c|c|c|c|c|c|c|c|c|c|}
\hline \multirow[t]{2}{*}{ Treatments } & \multicolumn{2}{|c|}{$\begin{array}{c}\text { Total } \\
\text { chlorophyll } \\
\text { content }\end{array}$} & \multicolumn{2}{|c|}{ Heading date } & \multicolumn{2}{|c|}{ Maturity date } & \multicolumn{2}{|c|}{ Plant height } & \multicolumn{2}{|c|}{$\begin{array}{l}\text { Number of } \\
\text { spikes } / \mathrm{m}^{2}\end{array}$} & \multicolumn{2}{|c|}{ Spike length } \\
\hline & S1 & S2 & $S_{1}$ & $\mathrm{~S}_{2}$ & $S_{1}$ & $\mathrm{~S}_{2}$ & $\mathrm{~S}_{1}$ & $\mathrm{~S}_{2}$ & $S_{1}$ & $\mathrm{~S}_{2}$ & S1 & S2 \\
\hline Normal & 44.49 & 44.36 & 88.00 & 90.53 & 126.70 & 133.50 & 108.63 & 112.43 & 457.80 & 446.40 & 7.33 & 9.37 \\
\hline Stress & 47.22 & 44.79 & 83.33 & 89.13 & 120.77 & 129.13 & 90.40 & 100.97 & 409.60 & 332.13 & 7.13 & 9.10 \\
\hline F-Test & ns & ns & * & NS & ** & ** & ** & * & ** & ** & ns & Ns \\
\hline $\mathrm{LSD}_{0.05}$ & & & 2.31 & - & - & - & - & 5.89 & - & - & & \\
\hline $\mathrm{LSD}_{0.01}$ & & & - & - & 2.39 & 3.82 & 12.71 & - & 31.01 & 32.44 & & \\
\hline
\end{tabular}

NS, ${ }^{*}$ and ${ }^{* *}$ indicated not significant, significant at 0.05 and significant at 0.01 levels of probability, respectively.

Table (4 b): Effect of irrigation treatments on barley characters in both growing seasons

\begin{tabular}{|c|c|c|c|c|c|c|c|c|c|c|}
\hline \multirow{2}{*}{ Treatments } & \multicolumn{2}{|c|}{$\begin{array}{c}\text { Number of } \\
\text { grains/spike }\end{array}$} & \multicolumn{2}{|c|}{$\begin{array}{c}\text { 1000-grain } \\
\text { weight (g) }\end{array}$} & \multicolumn{2}{|c|}{$\begin{array}{c}\text { Biological } \\
\text { yield } \\
\text { (ton/fed.) }\end{array}$} & \multicolumn{2}{|c|}{$\begin{array}{c}\text { Grain yield } \\
\text { (ton/fed) }\end{array}$} & \multicolumn{2}{|c|}{$\begin{array}{c}\text { Harvest } \\
\text { index }\end{array}$} \\
\cline { 2 - 12 } & $\mathrm{S} 1$ & $\mathrm{~S} 2$ & $\mathrm{~S}_{1}$ & $\mathrm{~S}_{2}$ & $\mathrm{~S}_{1}$ & $\mathrm{~S}_{2}$ & $\mathrm{~S}_{1}$ & $\mathrm{~S}_{2}$ & $\mathrm{~S} 1$ & $\mathrm{~S} 2$ \\
\hline Normal & 51.10 & 70.00 & 51.01 & 49.83 & 8.48 & 12.52 & 3.44 & 4.55 & 40.62 & 37.04 \\
\hline Stress & 50.60 & 66.40 & 46.84 & 47.89 & 5.52 & 7.47 & 2.42 & 2.97 & 44.12 & 39.88 \\
\hline F-Test & $\mathrm{ns}$ & $\mathrm{ns}$ & $\mathrm{NS}$ & $*$ & $* *$ & $* *$ & $* *$ & $* *$ & $\mathrm{~ns}$ & $\mathrm{~ns}$ \\
\hline $\mathrm{LSD}_{0.05}$ & & & - & 1.41 & - & - & - & - & & \\
\hline $\mathrm{LSD}_{0.01}$ & & & - & - & 1.72 & 2.19 & 0.58 & 0.12 & & \\
\hline
\end{tabular}




\section{Effect of barley genotypes:}

The results in Table (5) showed that, all the ten studied genotypes were significantly different in all the studied characters in both seasons, except harvest index in the first season. The all results for all the studied characters were not similar in the two seasons of study. G 134 recorded the highest values for total chlorophyll content in the first season, while, the highest value in the second seasons found by $\mathrm{G} 133$. On the other hand, there were no difference effects between $\mathrm{G} 133$ and $\mathrm{G} 134$ in both seasons. The lowest values of heading date towards earliness were showed with $G 123$ and $G$ 134 in the first and second seasons, respectively. While, the lowest values of maturity date recorded by $G 133$ in first season and $G 123$ and $G 129$ in the second season, as shown as in Table (5). The results showed the earlier genotypes received less water than the later ones, thus reducing the drought escape effect and the advantages of earliness with respect to field conditions. All genotypes were earlier than Giza 136 and Giza 135 in the first and second seasons, which they needed longer time to reach maturity. With respect to plant height, the results showed most genotypes were taller than Giza 133, especially Giza132 which showed the superiority in both seasons. Giza 131 and Giza 132 had highest values for spike length in first and second seasons, respectively. For grains number per spike, Giza131 and Giza 133 had higher values in the first and second seasons, respectively, as shown as in Table (5).

In Table (6) showed that, Giza 133 recorded the highest values of spikes number $/ \mathrm{m}^{2}$, biological yield and grain yield/fed. in both seasons. While, 1000-grain weight, most genotypes had higher values compared with Giza 129 in both seasons, especially Giza 123 in both seasons. The highest values of harvest index found with Giza 136 in both seasons, while the lowest values were recorded with Giza 132 and Giza 131 in the first and the second seasons.

These results may be due to genetical variation. Generally, water stress reduced plant height, number of spikes $/ \mathrm{m}^{2}$ by reducing number of tillers, grain weight, spikelets/ spike and grain yield/ha (Samarah, 2005). These results go in line with those reported by Kheiralla et al. (1997), Tarred et al., (2002), Motawei and Abdalla (2003), El-Kholy et al., (2005), Karami et al., (2005) and Abu-El-Lail et al., (2016).

\section{Effect of the interaction between barley genotypes and irrigation treatments.}

The data in Table (7 a and b) showed that the interaction between barley genotypes and irrigation treatments for all the studied characters.

In the first season, significant interaction between barley genotypes and irrigation treatments was found in most characters, except for spike length, no. of grains/spike and no. of spike $/ \mathrm{m}^{2}$ in both seasons and plant height and harvest index in first season, as shown as in Table (7 a and b). However, it seems that these genotypes affected by changing environments.

In both seasons, significant and highly significant interaction between barley genotypes, irrigation conditions and seasons were found in most characters, except plant height and harvest index in the first season.

This would indicate that, genotypes differ with the change of water condition and revealing that the performance of genotypes differ with the change of seasons.

On the other hand, the highest values of total chlorophyll content were found by Giza 134 and Giza 133 in the first and second seasons under drought stress, respectively. Giza 123 under stress recorded the earliest 


\section{E. H. El-Seidy, et al.,}

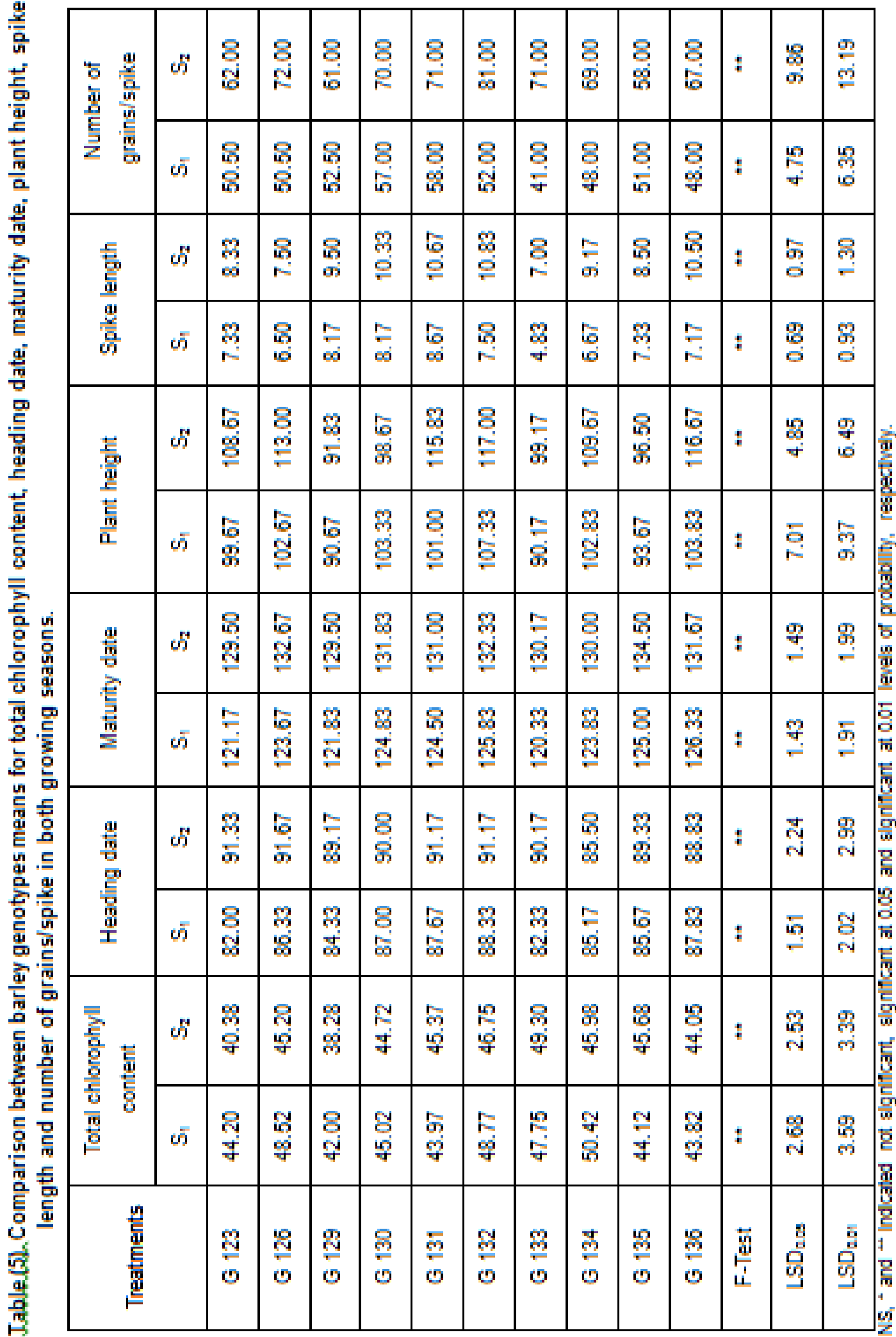




\begin{tabular}{|c|c|c|c|c|c|c|c|c|c|c|c|c|c|c|}
\hline 范高 & $\tilde{\omega}^{4}$ & $\begin{array}{l}8 \\
\text { กิ }\end{array}$ & $\stackrel{8}{\stackrel{8}{N}}$ & $\frac{8}{\dot{0}}$ & 吕 & $\frac{8}{1}$ & $\frac{8}{\infty}$ & $\frac{8}{1}$ & $\begin{array}{l}8 \\
\text { ஜ் }\end{array}$ & $\begin{array}{l}8 \\
0 \\
0\end{array}$ & 号 & ॠ & $\begin{array}{l}\mathscr{\infty} \\
\infty \\
\sigma\end{array}$ & 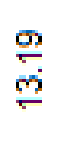 \\
\hline 竞 高 & ம் & 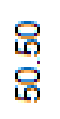 & 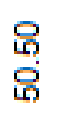 & $\begin{array}{l}\text { ํํํ } \\
\text { त̂ }\end{array}$ & 8 & $\begin{array}{l}8 \\
0 \\
00 \\
60\end{array}$ & $\begin{array}{l}80 \\
\text { กิ }\end{array}$ & $\frac{8}{\frac{8}{4}}$ & $\begin{array}{l}8 \\
\text { o } \\
\text { q }\end{array}$ & $\frac{8}{50}$ & $\begin{array}{l}8 \\
\text { o } \\
\text { q }\end{array}$ & 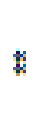 & 号 & $\stackrel{\leftrightarrow}{\mathrm{m}}$ \\
\hline $\begin{array}{l}\text { 도 } \\
\text { 듬 } \\
\text { 던 }\end{array}$ & $\delta^{4}$ & $\stackrel{m}{m}$ & 品 & $\begin{array}{l}\text { 吕 } \\
\text { ஸे }\end{array}$ & mे & $\begin{array}{l}\stackrel{ }{\circ} \\
\stackrel{0}{\circ}\end{array}$ & $\begin{array}{l}\text { OO } \\
\text { 웅 }\end{array}$ & 8 & $\stackrel{5}{\sigma}$ & $\underset{\infty}{0}$ & $\begin{array}{l}\text { 웅 } \\
\stackrel{0}{0}\end{array}$ & 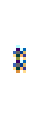 & Бे & ભి \\
\hline $\begin{array}{l}\text { 崩 } \\
\text { के }\end{array}$ & ஸ் & m & 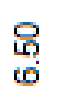 & $\underset{\infty}{\approx}$ & $\stackrel{5}{\infty}$ & $\begin{array}{l}n \\
\infty \\
\infty\end{array}$ & 星 & $\begin{array}{l}\text { MO } \\
\text { \& } \\
\dot{\nabla}\end{array}$ & $\begin{array}{l}\hat{c} \\
\stackrel{\omega}{0}\end{array}$ & m̧ & $\stackrel{5}{\pi}$ & 象 & $\stackrel{8}{\circ}$ & $\stackrel{8}{\circ}$ \\
\hline 蒿 & $\tilde{\omega}^{4}$ & $\begin{array}{l}\text { 음 } \\
\circ \\
\circ\end{array}$ & $\begin{array}{l}8 \\
\stackrel{\text { ji }}{=}\end{array}$ & ஜ & $\begin{array}{l}\hat{\circ} \\
\circ \\
\circ\end{array}$ & 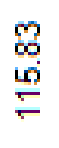 & $\begin{array}{l}8 \\
\stackrel{5}{=}\end{array}$ & $\stackrel{5}{\circ}$ & $\begin{array}{l}\text { ㄴo } \\
\text { த் }\end{array}$ & $\begin{array}{l}\text { 웅 } \\
\stackrel{\circ}{\circ}\end{array}$ & 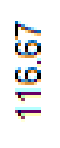 & 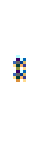 & $\begin{array}{l}\stackrel{2}{\infty} \\
\stackrel{0}{+}\end{array}$ & 守 \\
\hline $\begin{array}{l}\overrightarrow{\vec{c}} \\
\frac{\bar{\sigma}}{\bar{\alpha}}\end{array}$ & ஸ் & $\begin{array}{l}\text { 苗 } \\
\text { ळ }\end{array}$ & $\begin{array}{l}\text { 으 } \\
\text { ợ }\end{array}$ & $\begin{array}{l}\text { 他 } \\
\text { के }\end{array}$ & $\begin{array}{l}m \\
\text { m. }\end{array}$ & 웅 & $\begin{array}{l}m \\
\stackrel{3}{\circ}\end{array}$ & 홍 & 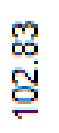 & 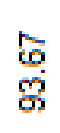 & $\begin{array}{l}3 \\
\infty \\
\dot{8} \\
\end{array}$ & 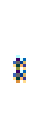 & ס & ले \\
\hline 荡 & $\tilde{\delta}^{4}$ & 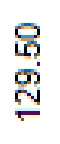 & ڤึ & 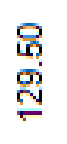 & 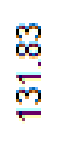 & $\stackrel{8}{\stackrel{m}{-}}$ & लू & $\stackrel{\circ}{\check{D}}$ & 욤 & $\begin{array}{l}\text { 品 } \\
\text { m }\end{array}$ & 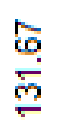 & 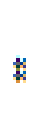 & $\stackrel{g}{\text { g }}$ & 。ํ \\
\hline $\begin{array}{l}\text { 莺 } \\
\sum\end{array}$ & ஸ் & $\stackrel{ }{\stackrel{丶}{\leftrightarrows}}$ & 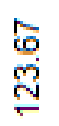 & 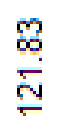 & 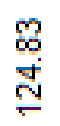 & $\begin{array}{l}\text { 웅 } \\
\stackrel{\mathrm{d}}{\mathrm{O}}\end{array}$ & 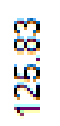 & लై & $\begin{array}{l}\mathscr{M} \\
\stackrel{\text { }}{\sigma}\end{array}$ & 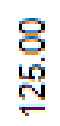 & 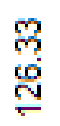 & 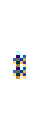 & $\stackrel{\text { ģ }}{-}$ & $\stackrel{5}{\circ}$ \\
\hline $\begin{array}{l}\text { 湈 } \\
\text { व } \\
\text { 임 }\end{array}$ & $\tilde{\omega}$ & लై & চ்̋ & $\stackrel{r}{\check{\infty}}$ & $\begin{array}{l}8 \\
8 \\
8\end{array}$ & $\frac{1}{5}$ & $\frac{5}{5}$ & $\frac{1}{8}$ & $\begin{array}{l}\text { 昂 } \\
\text { ஸึ } \\
\infty\end{array}$ & लె & $\begin{array}{l}\mathscr{M} \\
\infty \\
\infty \\
\infty\end{array}$ & 象 & $\stackrel{\sim}{N}$ & $\stackrel{\text { g }}{\mathrm{N}}$ \\
\hline 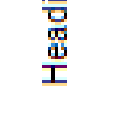 & ஸे & $\begin{array}{l}8 \\
\text { ભ }\end{array}$ & m్ & m్ & $\begin{array}{l}8 \\
\text { 心 }\end{array}$ & 灾 & $\begin{array}{l}m \\
\infty \\
\infty \\
\infty\end{array}$ & ભై & $\stackrel{ }{\frac{1}{\infty}}$ & $\begin{array}{l}n \\
0 \\
0 \\
\infty\end{array}$ & 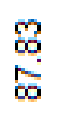 & 象 & $\stackrel{\overline{6}}{\stackrel{\circ}{-}}$ & $\underset{\text { ¿ }}{\text { ¿ }}$ \\
\hline 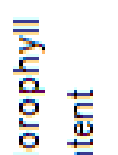 & ஸึ & 啇 & $\begin{array}{l}\text { 오 } \\
\text { ษั }\end{array}$ & 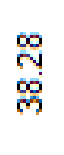 & $\begin{array}{l}\mathbb{N} \\
\text { J }\end{array}$ & $\begin{array}{l}\text { ले } \\
\text { 岁 }\end{array}$ & $\begin{array}{l}\frac{2}{2} \\
6 \\
6 \\
o\end{array}$ & 命 & $\begin{array}{l}\text { \% } \\
\text { \% } \\
\text { 守 }\end{array}$ & $\begin{array}{l}\stackrel{8}{0} \\
\stackrel{\circ}{9}\end{array}$ & $\begin{array}{l}\text { 号 } \\
\text { 寸 }\end{array}$ & * & $\stackrel{\mathscr{0}}{\mathrm{N}}$ & $\begin{array}{l}\text { ले } \\
\text { ले }\end{array}$ \\
\hline $\begin{array}{l}\frac{\overline{0}}{0} \\
\frac{\pi}{50} \\
\frac{0}{\circ}\end{array}$ & ஸ் & సิ & $\begin{array}{l}\text { గ్ } \\
\text { ơ } \\
\text { o }\end{array}$ & $\begin{array}{l}8 \\
\text { ชิ }\end{array}$ & $\begin{array}{l}\text { ठั } \\
\text { gु }\end{array}$ & $\begin{array}{l}\text { के } \\
\text { ले }\end{array}$ & $\begin{array}{l}E \\
\text { o } \\
\text { o }\end{array}$ & \begin{tabular}{l}
$\stackrel{2}{2}$ \\
\multirow{2}{*}{}
\end{tabular} & 芒 & $\frac{\mathfrak{Z}}{\square}$ & $\begin{array}{l}\text { ळ } \\
\text { ஜु }\end{array}$ & 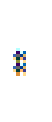 & $\stackrel{\circ}{\circ}$ & 只 \\
\hline 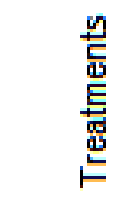 & & $\underset{0}{\frac{M}{6}}$ & $\stackrel{\mathscr{O}}{\underset{0}{0}}$ & $\underset{0}{\frac{\mathbb{S}}{0}}$ & $\frac{\text { 웅 }}{0}$ & $\frac{\bar{m}}{0}$ & $\stackrel{\text { लै }}{\circ}$ & $\frac{m}{-1}$ & $\frac{\text { ले }}{\sigma}$ & $\frac{\text { m }}{0}$ & $\frac{\mathscr{m}}{\circ}$ & 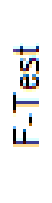 & 总 & 몽 \\
\hline
\end{tabular}


E. H. El-Seidy, et al.,

Table (7 a). Effect of the interaction between barley genotypes and irrigation treatment on all studied characters in both growing seasons.

\begin{tabular}{|c|c|c|c|c|c|c|c|c|c|c|c|c|}
\hline \multirow[t]{2}{*}{ Treatments } & \multicolumn{2}{|c|}{$\begin{array}{c}\text { Total } \\
\text { chlorophyll } \\
\text { content }\end{array}$} & \multicolumn{2}{|c|}{$\begin{array}{l}\text { Heading } \\
\text { date }\end{array}$} & \multicolumn{2}{|c|}{ Maturity date } & \multicolumn{2}{|c|}{ Plant height } & \multicolumn{2}{|c|}{$\begin{array}{l}\text { Number of } \\
\text { spikes } / \mathrm{m}^{2}\end{array}$} & \multicolumn{2}{|c|}{ Spike length } \\
\hline & $S_{1}$ & $\mathrm{~S}_{2}$ & $S_{1}$ & $\mathrm{~S}_{2}$ & $\mathrm{~S}_{1}$ & $\mathrm{~S}_{2}$ & $\mathrm{~S}_{1}$ & $\mathrm{~S}_{2}$ & $S_{1}$ & $\mathrm{~S}_{2}$ & $S_{1}$ & $\mathrm{~S}_{2}$ \\
\hline
\end{tabular}

Normal irrigation conditions

\begin{tabular}{|c|c|c|c|c|c|c|c|c|c|c|c|c|}
\hline G 123 & 44.37 & 41.93 & 83.67 & 93.00 & 123.00 & 132.33 & 112.00 & 118.67 & 436.00 & 430.00 & 7.33 & 8.33 \\
\hline G 126 & 45.93 & 44.33 & 88.00 & 95.00 & 127.33 & 135.67 & 114.00 & 118.33 & 450.00 & 468.00 & 7.00 & 7.33 \\
\hline G 129 & 43.67 & 40.73 & 87.67 & 88.67 & 122.67 & 131.00 & 94.67 & 97.33 & 490.00 & 432.00 & 7.67 & 10.33 \\
\hline G 130 & 44.63 & 42.13 & 90.00 & 88.67 & 128.67 & 133.33 & 113.00 & 102.67 & 540.00 & 518.00 & 5.00 & 7.33 \\
\hline G 131 & 42.83 & 46.43 & 92.00 & 92.33 & 129.33 & 131.67 & 108.33 & 115.33 & 504.00 & 518.00 & 6.00 & 9.33 \\
\hline G 132 & 43.80 & 44.67 & 89.67 & 93.33 & 129.33 & 136.67 & 116.33 & 126.33 & 414.00 & 390.00 & 8.00 & 9.67 \\
\hline G 133 & 46.30 & 49.17 & 84.00 & 93.00 & 123.00 & 131.67 & 97.67 & 100.67 & 434.00 & 452.00 & 8.67 & 11.00 \\
\hline G 134 & 45.50 & 45.60 & 86.33 & 82.33 & 126.33 & 132.33 & 114.33 & 114.33 & 442.00 & 452.00 & 8.67 & 10.67 \\
\hline G 135 & 44.27 & 47.00 & 89.00 & 91.67 & 128.67 & 137.00 & 102.67 & 103.33 & 466.00 & 422.00 & 7.33 & 9.33 \\
\hline G 136 & 43.60 & 41.57 & 89.67 & 87.33 & 128.67 & 133.33 & 113.33 & 127.33 & 402.00 & 382.00 & 7.67 & 10.33 \\
\hline
\end{tabular}

Water stress conditions

\begin{tabular}{|c|c|c|c|c|c|c|c|c|c|c|c|c|}
\hline G 123 & 44.03 & 38.83 & 80.33 & 89.67 & 119.33 & 126.67 & 87.33 & 98.67 & 402.00 & 356.00 & 7.33 & 8.33 \\
\hline G 126 & 51.10 & 46.07 & 84.67 & 88.33 & 120.00 & 129.67 & 91.33 & 107.67 & 414.00 & 324.00 & 6.00 & 7.67 \\
\hline G 129 & 40.33 & 35.83 & 81.00 & 89.67 & 121.00 & 128.00 & 86.67 & 86.33 & 410.00 & 336.67 & 7.33 & 11.33 \\
\hline G 130 & 45.40 & 47.30 & 84.00 & 91.33 & 121.00 & 130.33 & 93.67 & 94.67 & 456.00 & 374.00 & 4.67 & 6.67 \\
\hline G 131 & 45.10 & 44.30 & 83.33 & 90.00 & 119.67 & 130.33 & 93.67 & 116.33 & 452.00 & 364.00 & 7.33 & 9.00 \\
\hline G 132 & 53.73 & 48.83 & 87.00 & 89.00 & 122.33 & 128.00 & 98.33 & 107.67 & 378.00 & 280.67 & 8.33 & 9.33 \\
\hline G 133 & 49.20 & 49.43 & 80.67 & 87.33 & 117.67 & 128.67 & 82.67 & 97.67 & 462.00 & 298.00 & 7.67 & 9.67 \\
\hline G 134 & 55.33 & 46.37 & 84.00 & 88.67 & 121.33 & 127.67 & 91.33 & 105.00 & 342.00 & 344.00 & 8.67 & 10.67 \\
\hline G 135 & 43.97 & 44.37 & 82.33 & 87.00 & 121.33 & 132.00 & 84.67 & 89.67 & 380.00 & 354.00 & 7.33 & 7.67 \\
\hline G 136 & 44.03 & 46.53 & 86.00 & 90.33 & 124.00 & 130.00 & 94.33 & 106.00 & 400.00 & 290.00 & 6.67 & 10.67 \\
\hline F-Test & $* *$ & ** & $* *$ & ** & $* *$ & $* *$ & NS & $* *$ & ns & ns & ns & ns \\
\hline $\mathrm{LSD}_{0.05}$ & 3.80 & 3.58 & 2.14 & 3.16 & 2.02 & 2.11 & - & 6.86 & & & & \\
\hline $\mathrm{LSD}_{0.01}$ & 5.08 & 4.79 & 2.86 & 4.23 & 2.70 & 2.82 & - & 9.18 & & & & \\
\hline
\end{tabular}

NS, ${ }^{*}$ and ${ }^{* *}$ indicated not significant, significant at 0.05 and significant at 0.01 levels of probability, respectively. 
Evaluation of ten barley genotypes under normal and drought stress

Table (7 b). Effect of the interaction between barley genotypes and irrigation treatment on all studied characters in both growing seasons.

\begin{tabular}{|c|c|c|c|c|c|c|c|c|c|c|}
\hline \multirow{2}{*}{ Treatments } & \multicolumn{2}{|c|}{$\begin{array}{l}\text { Number of } \\
\text { grains/spike }\end{array}$} & \multicolumn{2}{|c|}{$\begin{array}{l}\text { 1000-grain } \\
\text { weight }(\mathrm{g})\end{array}$} & \multicolumn{2}{|c|}{$\begin{array}{c}\text { Biological } \\
\text { yield (ton/fed.) }\end{array}$} & \multicolumn{2}{|c|}{$\begin{array}{c}\text { Grain yield } \\
\text { (ton/fed.) }\end{array}$} & \multicolumn{2}{|c|}{ Harvest index } \\
\hline & S1 & S2 & S1 & S2 & S1 & S2 & S1 & $\mathrm{S} 2$ & S1 & S2 \\
\hline \multicolumn{11}{|c|}{ Normal irrigation conditions } \\
\hline G 123 & 51.00 & 62.00 & 55.07 & 55.79 & 8.55 & 10.92 & 3.53 & 4.57 & 41.38 & 41.95 \\
\hline G 126 & 53.00 & 72.00 & 50.47 & 50.46 & 8.23 & 11.86 & 3.23 & 4.55 & 39.46 & 38.30 \\
\hline G 129 & 52.00 & 80.00 & 43.37 & 43.47 & 7.88 & 10.11 & 3.27 & 4.20 & 41.64 & 41.78 \\
\hline G 130 & 44.00 & 72.00 & 47.80 & 45.57 & 8.50 & 11.57 & 3.75 & 4.43 & 44.08 & 38.54 \\
\hline G 131 & 46.00 & 70.00 & 51.93 & 45.03 & 8.62 & 12.89 & 3.27 & 4.39 & 37.93 & 34.22 \\
\hline G 132 & 51.00 & 70.00 & 57.97 & 51.83 & 8.96 & 13.78 & 3.32 & 4.36 & 37.11 & 32.05 \\
\hline G 133 & 60.00 & 72.00 & 50.63 & 53.93 & 8.71 & 15.22 & 3.44 & 4.81 & 39.45 & 31.88 \\
\hline G 134 & 56.00 & 68.00 & 53.10 & 51.60 & 8.89 & 14.63 & 3.70 & 4.85 & 41.66 & 33.18 \\
\hline G 135 & 50.00 & 66.00 & 47.17 & 44.63 & 8.45 & 13.48 & 3.32 & 4.61 & 39.29 & 34.26 \\
\hline G 136 & 48.00 & 68.00 & 52.60 & 56.00 & 8.06 & 10.70 & 3.55 & 4.73 & 44.16 & 44.27 \\
\hline \multicolumn{11}{|c|}{ Water stress conditions } \\
\hline G 123 & 50.00 & 62.00 & 52.37 & 53.27 & 4.70 & 7.47 & 2.09 & 2.94 & 44.93 & 39.32 \\
\hline G 126 & 48.00 & 72.00 & 49.00 & 50.50 & 5.93 & 7.65 & 2.66 & 3.21 & 45.46 & 41.98 \\
\hline G 129 & 52.00 & 82.00 & 41.17 & 37.40 & 4.50 & 6.09 & 1.90 & 2.03 & 42.17 & 33.70 \\
\hline G 130 & 38.00 & 70.00 & 49.00 & 44.30 & 5.88 & 7.77 & 2.49 & 2.74 & 42.15 & 35.37 \\
\hline G 131 & 50.00 & 68.00 & 45.97 & 44.70 & 5.95 & 8.59 & 2.59 & 3.12 & 43.70 & 37.41 \\
\hline G 132 & 54.00 & 52.00 & 44.37 & 50.40 & 6.12 & 7.97 & 2.56 & 3.34 & 41.88 & 42.06 \\
\hline G 133 & 54.00 & 68.00 & 50.47 & 54.70 & 6.59 & 8.47 & 2.94 & 3.71 & 44.67 & 44.37 \\
\hline G 134 & 60.00 & 74.00 & 46.87 & 52.60 & 6.58 & 7.79 & 2.84 & 3.36 & 43.09 & 43.31 \\
\hline G 135 & 52.00 & 50.00 & 41.53 & 38.47 & 4.02 & 6.63 & 1.90 & 2.51 & 47.22 & 37.97 \\
\hline G 136 & 48.00 & 66.00 & 47.67 & 52.60 & 4.91 & 6.27 & 2.25 & 2.71 & 45.91 & 43.30 \\
\hline F-Test & ns & ns & ** & ** & * & * & * & ** & NS & ** \\
\hline LSD0.05 & & & 4.88 & 2.43 & 0.90 & 1.72 & 0.49 & 0.10 & - & 6.59 \\
\hline LSD0.01 & & & 6.52 & 3.25 & - & - & - & 0.13 & - & 8.82 \\
\hline
\end{tabular}

NS, ${ }^{*}$ and ${ }^{* *}$ indicated not significant, significant at 0.05 and significant at 0.01 levels of probability, respectively. 
heading date in the first season, while Giza 134 under normal irrigation recorded the earliest heading date in the second season. Giza 123 under stress condition was the earliest genotypes for maturity date in both seasons. The highest plant height showed by Giza 132 in both seasons under normal irrigation conditions. On the other hand, Giza 132 under normal irrigation condition showed the highest values of 1000-grain weight in the first season and Giza 136 in the second season under the same irrigation method, as shown as in Table ( $7 \mathrm{a}$ and $b$ ).

The highest grain yield (ton/fed.) was recorded by the interactions between normal irrigation and genotype Giza 134 and 130 in the first season and Giza 134 and Giza 133 in the second season under normal irrigation conditions. While, the lowest grain yield was recorded by Giza 129 and Giza 135 in the first season and Giza 129 in the second season under the drought stress conditions. On the other hand, Giza 132 and Giza 133 were the superiority genotypes for biological yield in the first and second seasons under the normal irrigation treatments, respectively. For harvest index, Giza 133 and Giza 134 recorded the highest values with the interaction stress and normal irrigation, respectively in the second season, as shown as in Table ( $7 \mathrm{a}$ and $\mathrm{b}$ ).

As a result of water stress condition, the average of grain yield for these genotypes decreased. Several authors reported that, drought stress reduced photosynthesis and translocation rates and increased respiration, which reduced available assimilates for grain filling and finally decreased grain yield (El- Naggar, 2010 and Zare et al., 2011).

This study showed that, the best genotypes of barley for all parameters studied under drought conditions were Giza 133 followed by Giza 134 in both seasons, while, the lowest barely genotypes were found by Giza 126 and Giza 135 in both seasons.
Finally, from the present results under the same conditions we can recommended that, Giza 133 or Giza 134 under drought condition obtained that highest grain yield/fad.

\section{REFERENCES}

Abu-El-Lail, F.F.B., K.A. Hamam, K.A. Kheiralla and M.Z. El-Hifny (2016). Evaluation of Twenty Barley Genotypes For Drought Tolerance Under Sandy Clay Soil Egypt. J. Agron, 38 (2): 173187.

Ashraf. M. (2010). Inducing drought tolerance in plants: Recent Advances, Biotech. Adv. 28: 169-183.

Assey, A.A., M.E. Saleh, I.E. Ramadan and A.A. El-Sayed (1990). Effect of irrigation and applying nitrogen on barley. Zagazig J. Agric. Res. 17 (3A), 613-622.

Ceccarelli, S. (1986). Tolerance to climatic stresses. Proceed. 5th Int. Barley Genetic Symposium, Okayama 6-12 OCT.

El-Kholy, M.A., M.S. Gaballah, S. El-Ashry and A.M. El-Bawab (2005). Combating drought using yield stabilizing agents in barley. International J. Agric Biology. Pakistan, 7 (3), 369-375.

El-Madidi, S., Zineb Diani and F.B. Aameur (2005). Variation of agro-morphological characters in Moroccan barley landraces under near optimal and drought conditions. Genetic Resources and Crop Evolution. Kluwer Academic Publishers, Dordrecht, Netherlands: 52 (7), 831-838.

El-Naggar, A.A.E.A. (2010). Genetical studies on drought tolerance of barley. M.Sc. Thesis, Fac. of Agric., Tanta Univ., Egypt.

El-Seidy, E.H. (1997). Estimation of genetic effects for earliness and some agronomic traits in barley under water stress and non-stress condition. Annals. of Agric. Sci., Zagazig Univ., Egypt , 35 (3), 11471164.

El-Shawy, E. E., Kh. A. Amer, E.H.E. ElSeidy and A.A. El Gammaal (2013). Growth Analysis and Yield Response of 
Barley as Affected by Irrigation Regimes Egypt. J. Agron.. 35 (1) : 1-19.

El-shawy, E.E.A (2013). Assessment of water stress Tolerance in selected burley genotypes. Ph.D . Thesis, Fac. Agric. Agronomy Dept., Tanta Univ.

Farshadfar, E. and J. Sutka (2002). Screening drought tolerance criteria in maize. Acta. Agron. Hungarica, 50:411416.

Gaspar, I., E. Zama and C. Drobota (1998). Modifications caused by weather stress in the morpho-productive elements of spring barley. Cercetari Agronomice in Moldova,31 (1/2), 67-71.

Gomez, K.A. and A. Gomez (1984). Statistical procedures of Agricultural Research. John Wiley \& Sons, New York, 2nd ed., 680p.

Karami, E., M.R. Ghannadha, M.R. Naghavi and M. Mardi (2005). An evaluation of drought resistance in barley. Iranian J. Agric. Sci. Fac. Agric, Univ of Tehran, Iran, 36 (3), 547-560.

Kherialla, K.A., A.A. Ismail and G.R. ElNagar (1997). Drought tolerance and stability of some spring wheat cultivars. Assiut J. Agric. Sci. 28, 75-88.
Motawei, M.I. and M.Y. Abdalla (2003). Selection of resistance to Fusarium germinerum in barley under water stress by DNA markers. Alex. J. Agric. Res. 48 (1), 11-20.

Robins, J.S. and C.E. Domingo (1962). Moisture and nitrogen effects on irrigated spring wheat. Agron. J. 54 (1), 135-138

Samarah, N.H. (2005). Effects of drought stress on growth and yield of barley. Agron for Sustainable Development. EDP Sciences, Les Ulis, France, 25 (1), 145149.

Tarred, A.M., A.M. Megahed and Fatmaa Abdo (2002). Effect of irrigation intervals on some physiological and yield traits of barley under sprinkler irrigation system. Zagazig J. Agric. Res. 29 (3), 877-890.

Waller, R.A. and D.B. Duncan (1969). Abays rule for the symmetric multiple comparison problems. Amer. State. Assoc. J. Des. 1458-1503.

Zare, M., M.H. Azizi and F. Bazrafshan (2011). Effect of drought stress on some agronomic traits in ten barley (Hordeum vulgar, L.) cultivars. Tech. J. Engin. \& App. Sci., 1 (3), 57-62. 


\section{تقييم عشرة تراكيب وراثية من الشعير تحت ظروف الرى الطبيعي والاجهاد الرطويى}

السيد حامد الصعيدى(1) ، السيد السيد الثاوى(2) ، اسامة عبد الحميد عبدالرازق(1) ،

وليد جمعه الكردى (1)

(1) قسم المحاصيل كلية الزراعه جامعه طنطا

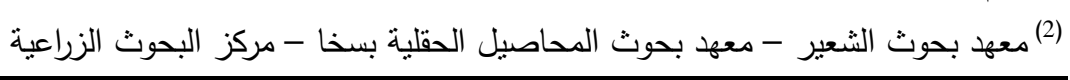

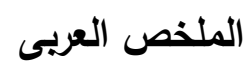

أجري هذا البحث بهدف التعرف على أفضل التراكيب الوراثية للشعبر التي يمكن زراعتها في ظل ظروف الإجهاد المائى.

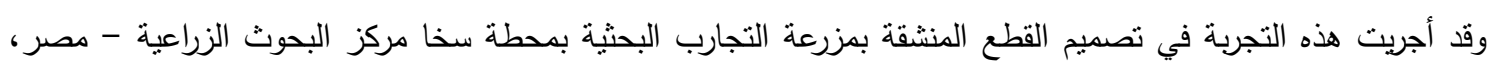

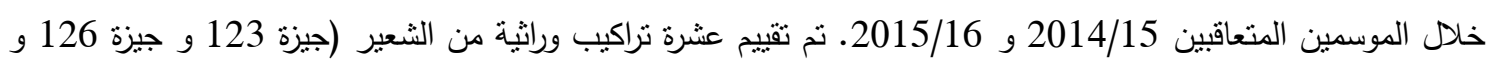

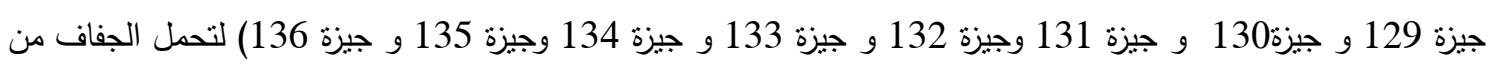

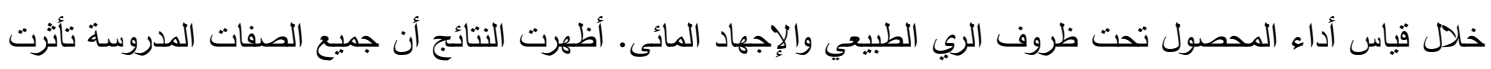

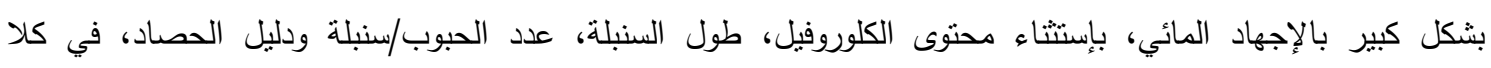
الموسمين. كما أدى الإجهاد المائى إلى انخفاض محصول الحبوب من خلال تقليل عدد السنابل/منز مربع ووزن الـ 1000

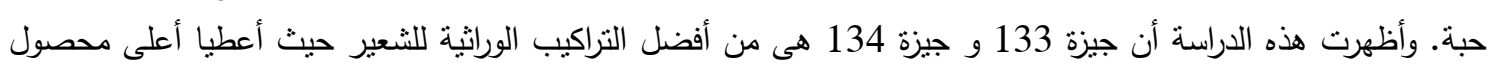

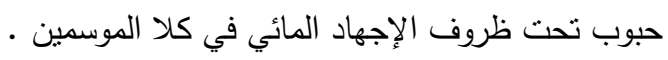

